\title{
Localization of gravity on a de Sitter thick braneworld without scalar fields
}

\author{
Alfredo Herrera-Aguilar, Dagoberto Malagón-Morejón², \\ and Refugio Rigel Mora-Luna ${ }^{3}$ \\ Instituto de Física y Matemáticas, Universidad Michoacana de San Nicolás de Hidalgo. \\ Edificio C-3, Ciudad Universitaria, C.P. 58040, Morelia, Michoacán, México.
}

\begin{abstract}
In this work we present a simple thick braneworld model that is generated by an intriguing interplay between a 5D cosmological constant with a de Sitter metric induced in the 3-brane without the inclusion of scalar fields. We show that $4 \mathrm{D}$ gravity is localized on this brane, provide analytic expressions for the massive KaluzaKlein (KK) fluctuation modes and also show that the spectrum of metric excitations displays a mass gap. We finally present the corrections to Newton's law due to these massive modes. This model has no naked singularities along the fifth dimension despite the existence of a mass gap in the graviton spectrum as it happens in thick branes with 4D Poincaré symmetry, providing a simple model with very good features: the curvature is completely smooth along the fifth dimension, it localizes $4 \mathrm{D}$ gravity and the spectrum of gravity fluctuations presents a mass gap, a fact that rules out the existence of phenomenologically dangerous ultralight KK excitations in the model. We finally present our solution as a limit of scalar thick branes.
\end{abstract}

\section{The model and its physical consistency}

Within the framework of the braneworld models embedded in a spacetime with extra dimensions and after the success of the thin brane models, where singularities are present at the position of the branes [1]-[6], in solving the mass hierarchy and 4D gravity localization problems, it has became a matter of interest to find smooth braneworld solutions. In some models, such solutions are obtained by introducing one or several scalar fields in the bulk [7]-[17] and the large variety of scalar fields that can be used to generate these models gives rise to different scenarios without singularities at the position of the branes. By following the line of smoothing out the brane configurations we show that $4 \mathrm{D}$ gravity can be localized on a de Sitter 3-brane generated in five dimensions by gravity with a positive cosmological constant only, avoiding at all the use of scalar fields. Thus, our thick brane is not made

\footnotetext{
${ }^{1}$ E-mail: herrera@ifm.umich.mx

${ }^{2}$ E-mail: malagon@ifm.umich.mx

${ }^{3}$ E-mail: topc@ifm.umich.mx
} 
of scalar matter but rather is modeled by an intriguing relation between the curvatures generated by the 5D and 4D cosmological constants.

In this scenario, the inclusion of the 5D cosmological constant renders a completely smooth 5D manifold in the presence of a mass gap in the KK graviton spectrum of metric fluctuations, in contrast with previous results found in the context of thick braneworlds with 4D Poincaré symmetry [8], [14].

The complete action for the braneworld model is expressed as follows

$$
S=2 M^{3} \int d^{5} x \sqrt{-g}\left(R-2 \Lambda_{5}\right) .
$$

It describes 5D gravity with a bulk cosmological constant $\Lambda_{5}$, and $M$ is the gravitational coupling constant in 5D (in fact we can write $\frac{1}{2 \kappa_{5}^{2}}$ instead of $2 M^{3}$ ).

The Einstein equations with a cosmological constant in five dimensions are given by

$$
G_{A B}=-\Lambda_{5} g_{A B}
$$

For the background metric we use the ansatz of a warped 5D line element with an induced 3-brane with spatially flat cosmological background that reads

$$
d s^{2}=e^{2 f(\sigma)}\left[-d t^{2}+a^{2}(t)\left(d x^{2}+d y^{2}+d z^{2}\right)\right]+d \sigma^{2}
$$

where $f(\sigma)$ is the warp factor and $a(t)$ is the scale factor of the brane.

By using the ansatz (3) we can compute the components of the Einstein tensor

$$
\begin{aligned}
G_{00} & =3\left[\frac{\dot{a}^{2}}{a^{2}}-e^{2 f}\left(2 f^{\prime 2}+f^{\prime \prime}\right)\right], \\
G_{\alpha \alpha} & =-2 \ddot{a} a-\dot{a}^{2}+3 a^{2} e^{2 f}\left(2 f^{\prime 2}+f^{\prime \prime}\right), \\
G_{\sigma \sigma} & =-3 e^{-2 f}\left(\frac{\ddot{a}}{a}+\frac{\dot{a}^{2}}{a^{2}}\right)+6 f^{\prime 2},
\end{aligned}
$$

where " " and ". " are derivatives with respect to the extra dimension and time, respectively.

The set of equations (2) can be rewritten in a simple way:

$$
\begin{aligned}
f^{\prime \prime} & =\frac{1}{3}\left(2 \frac{\dot{a}^{2}}{a^{2}}-5 \frac{\ddot{a}}{a}\right) e^{-2 f}, \\
f^{\prime 2} & =\frac{1}{6}\left[\left(5 \frac{\ddot{a}}{a}+\frac{\dot{a}^{2}}{a^{2}}\right)-\Lambda_{5}\right] e^{-2 f} .
\end{aligned}
$$

If we derive the restriction equation (6) with respect to the extra dimension in order to compare it to (5), we get a second order differential equation for the scale factor with a general solution of the following type

$$
a(t)=c e^{H t},
$$


where $c$ and $H$ are arbitrary constants. Thus, mathematical consistency of the Einstein equations forces us to have a scale factor corresponding to a de Sitter 4D cosmological background defined by $a(t)=e^{H t}$, since the constant $c$ can be absorbed into a coordinate redefinition. Once we have determined the form of the scale factor, the solution for the warp factor is straightforward and reads

$$
f(\sigma)=\ln \left(\frac{H}{b} \cos (b \sigma)\right)
$$

where $-\pi / 2 \leq \sigma \leq \pi / 2$, the constant $b$ is inversely proportional to the thickness of the 3 -brane and is determined by the $5 \mathrm{D}$ cosmological constant as follows:

$$
b^{2}=\frac{\Lambda_{5}}{6} .
$$

By performing the following coordinate transformation

$$
z=\int e^{-f(\sigma)} d \sigma
$$

the 5D metric (3) gets an overall conformal warp factor

$$
d s^{2}=e^{2 f(z)}\left[g_{\mu \nu} d x^{\mu} d x^{\nu}+d z^{2}\right]
$$

or, in terms of the metric $g_{M N}=e^{2 f} \bar{g}_{M N}$, which enables us to easily compute the 5D curvature scalar $R$ in terms of the $\bar{R}$ and the warp factor through the known formula [18]

$$
R=e^{-2 f}\left[\bar{R}-2(d-1) \bar{g}^{M N} \bar{\nabla}_{M} \bar{\nabla}_{N} f-(d-2)(d-1) g^{M N} \bar{\nabla}_{M} f \bar{\nabla}_{N} f\right],
$$

where $d$ is the total number of dimensions. This fact, in turn, allows us to separate an effective $4 \mathrm{D}$ action from the 5D one

$$
S_{e f f} \supset \int d^{4} x \sqrt{-\bar{g}}\left\{2 M^{3} \int_{-\infty}^{\infty}\left[e^{3 f} \bar{R}+4 H^{2} e^{3 f}\left(5 \operatorname{sech}^{2}(H z)-3\right)-2 e^{5 f} \Lambda_{5}\right] d z\right\} .
$$

After integrating over the extra coordinate $z(\sigma)$ we shall make a direct comparison of the result to the $4 \mathrm{D}$ Einstein-Hilbert action on the brane

$$
S_{\text {brane }}=2 M_{p l}^{2} \int d^{4} x \sqrt{-{ }^{4} g}\left({ }^{4} R-2 \Lambda_{4}\right) .
$$

Thus, in order to derive the scale of $4 \mathrm{D}$ gravitational interactions we look at the curvature term and perform the following integration:

$$
M_{P l}^{2}=M^{3} \int_{-\infty}^{\infty} e^{3 f(z)} d z=\frac{M^{3} H^{3}}{b^{3}} \int_{-\infty}^{\infty} \operatorname{sech}^{3}(H z) d z=\frac{\pi M^{3} H^{2}}{2 b^{3}}=\frac{3 \sqrt{6} \pi M^{3} H^{2}}{\Lambda_{5}^{3 / 2}}
$$

which turns out to be finite, as it should be for a well defined $4 \mathrm{D}$ theory. 
The second and third terms in the integral (12) contribute to the definition of the 4D cosmological constant $\Lambda_{4}$

$$
4 M_{p l}^{2} \Lambda_{4}=\int_{-\infty}^{\infty}\left[8 M^{3} H^{2} e^{3 f}\left(3-5 \operatorname{sech}^{2}(H z)\right)+4 M^{3} e^{5 f} \Lambda_{5}\right] d z .
$$

After integrating along the fifth dimension and simplifying the resulting expression we get

$$
\Lambda_{4}=3 H^{2}
$$

This relation can also be obtained from the Friedmann equations derived by directly varying the action (13).

\section{Metric perturbations, localization of $4 \mathrm{D}$ gravity and corrections to Newton's law}

In order to compute the corrections to Newton's law we need to perform some preliminary work. First of all we perturb the ansatz for the metric and then we compute the Einstein's equations to first order. For the sake of simplifying the calculations we use the freedom we have to impose an axial gauge $h_{5 M}=0$ on the perturbed metric:

$$
d s^{2}=e^{2 f(\sigma)}\left[g_{\mu \nu}+h_{\mu \nu}\right] d x^{\mu} d x^{\nu}+d \sigma^{2} .
$$

After imposing the transverse traceless condition $h_{\mu}^{\mu}=\partial^{\mu} h_{\mu \nu}=0$, the system of perturbed Einstein equations to first order adopts the form:

$$
\left[\partial_{\sigma}^{2}+4 f^{\prime} \partial_{\sigma}+e^{-2 f(\sigma)}\left(-\partial_{t}^{2}-3 H \partial_{t}+e^{-2 H t} \nabla^{2}-2 H^{2}\right)\right] \bar{h}_{\mu \nu}=0
$$

where $\nabla^{2}$ is the Laplacian in flat space and $\bar{h}_{\mu \nu}$ are the transverse traceless modes of the metric fluctuations.

In order to simplify calculations we perform the coordinate transformation (9) which allows us to study in detail the physics of the previous equation. Thus, the equation (18) takes the following form

$$
\left[\partial_{z}^{2}+3 f^{\prime} \partial_{z}-\partial_{t}^{2}-3 H \partial_{t}+e^{-2 H t} \nabla^{2}-2 H^{2}\right] \bar{h}_{\mu \nu}=0
$$

At this point we shall make use of the following separation of variables

$$
\bar{h}_{\mu \nu}=e^{-\frac{3}{2} f(z)} \Psi_{\mu \nu}(z) g(x) .
$$

This change of variables leads to a Schrödinger-like equation (where the indices of the function $\Psi_{\mu \nu}(z)$ have been omitted for convenience) for the 5D sector:

$$
\left(-\partial_{z}^{2}+\frac{9}{4} f^{\prime 2}+\frac{3}{2} f^{\prime \prime}-m^{2}\right) \Psi(z)=0
$$


where one can identify the quantum mechanical potential $V_{Q M}$ as follows

$$
V_{Q M}=\frac{9}{4} f^{\prime 2}+\frac{3}{2} f^{\prime \prime}
$$

Finally, the $4 \mathrm{D}$ equation that we obtain reads

$$
\left(-\partial_{t}^{2}-3 H \partial_{t}+e^{-2 H t} \nabla^{2}-2 H^{2}\right) g(x)=-m^{2} g(x),
$$

where the integration constant $m^{2}$ is defined as the mass in a 4D de Sitter spacetime [19].

The warp factor in the language of the variable $z$ is given by

$$
f=\ln \left(\frac{H}{b} \operatorname{sech}(H z)\right) .
$$

Thus, in terms of this coordinate, our quantum mechanical potential takes the form of a modified Pöschl-Teller one

$$
V_{Q M}=-\frac{15}{4} H^{2} \operatorname{sech}^{2}(H z)+\frac{9}{4} H^{2},
$$

which ensures the existence of a mass gap determined by its asymptotic value, namely, by $\frac{9}{4} H^{2}$, or equivalently $m=\frac{3 H}{2}$.

Thus, we have a classical eigenvalue problem for the following Schrödinger equation

$$
\left(-\partial_{z}^{2}-\frac{15}{4} H^{2} \operatorname{sech}^{2}(H z)+\frac{9}{4} H^{2}-m^{2}\right) \Psi(z)=0,
$$

with the general solution given by

$$
\Psi(z)=C_{1} P_{\frac{3}{2}}^{\mu}(\tanh (H z))+C_{2} Q_{\frac{3}{2}}^{\mu}(\tanh (H z))
$$

where $P_{\frac{3}{2}}^{\mu}$ and $Q_{\frac{3}{2}}^{\mu}$ are associated Legendre functions of first and second kind, respectively, of degree $\nu=3 / 2$ and order $\mu=\sqrt{\frac{9}{4}-\frac{m^{2}}{H^{2}}}[12]$.

Thus, the last differential equation has two discrete bound states: the first one corresponding to a massless bound state with energy $E_{0}=-\frac{9}{4} H^{2}$ and $\mu=3 / 2$

$$
\Psi_{0}(z)=k_{0} \operatorname{sech}^{\frac{3}{2}}(H z)
$$

physically interpreted as a stable graviton localized on the brane, and a second one corresponding to an excited state with $m^{2}=2 H^{2}$, energy $E_{1}=-\frac{1}{4} H^{2}$ and $\mu=\frac{1}{2}$

$$
\Psi_{1}(z)=k_{1} \sinh (H z) \operatorname{sech}^{\frac{3}{2}}(H z)
$$

which represents a massive graviton also localized on the brane; finally we have a tower of massive continuous modes that start from $m \geq \frac{3 H}{2}$ described by the following eigenfunctions with imaginary order $\mu=i \rho[12]$

$$
\Psi_{m}(z)=C_{1} P_{\frac{3}{2}}^{ \pm i \rho}(\tanh (H z))+C_{2} Q_{\frac{3}{2}}^{ \pm i \rho}(\tanh (H z))
$$


where $\rho=\sqrt{\frac{m^{2}}{H^{2}}-\frac{9}{4}}$, that must asymptote to plane waves.

This result prepares to us the way to compute corrections to Newton's law, since the massive modes contribute to Newton's law with small corrections coming from the fifth dimension. In order to find these corrections we consider $2 m>3 H$ and $C_{2}=0$ in equation (30) since the continuous spectrum of eigenfunctions asymptotically describes plane waves

$$
\Psi_{ \pm}^{\mu}(z)=C_{ \pm} P_{\frac{3}{2}}^{ \pm i \rho}(\tanh (H z)) \sim \frac{1}{\sqrt{2 \pi}} e^{ \pm i H \rho z} .
$$

After this, we further take the thin brane limit $H \longmapsto \infty$ and locate a probe mass in the center of the brane in the transverse direction. The corrections to the potential generated by massive gravitons can be expressed as follows [9]

$$
\begin{array}{r}
U(r) \sim \frac{M_{1} M_{2}}{r}\left(G_{4}+M_{*}^{-3} e^{-m_{1} r}\left|\Psi_{1}\left(z_{0}\right)\right|^{2}+M_{*}^{-3} \int_{m_{0}}^{\infty} d m e^{-m r}\left|\Psi^{\mu(m)}\left(z_{0}\right)\right|^{2}\right) \\
=\frac{M_{1} M_{2}}{r}\left(G_{4}+\triangle G_{4}\right)
\end{array}
$$

where the brane is located at $z=z_{0}, G_{4}$ is the gravitational coupling constant in $4 \mathrm{D}$, $\Psi_{1}\left(z_{0}\right)$ is the wave function of the first excited state and since it is an odd function, it does not contribute in the thin brane limit that we are considering, finally, $\Psi^{\mu}\left(z_{0}\right)$ represent the continuous massive states that need to be integrated over their masses. Thus, the corrections to Newton's law contributed by the massive continuous states have the following form (see [12] for details):

$$
\triangle G_{4} \sim M_{*}^{-3} \frac{1}{\left|\Gamma\left(-\frac{1}{4}\right) \Gamma\left(\frac{7}{4}\right)\right|^{2}} \frac{e^{-\frac{3}{2} H r}}{r}\left(1+O\left(\frac{1}{H r}\right)\right),
$$

indicating its small character.

It is worth mentioning that in our setup there also exists a relation between the ground bound state $\Psi_{0}(z)=k_{0} e^{\frac{3}{2} f(z)}$, which ensures $4 \mathrm{D}$ gravity localization, the smooth character of the 5D Ricci scalar $R$ and the behaviour of the quantum mechanical potential $V_{Q M}$ in the same spirit as it was done for flat thick 3-branes in [14], where it was shown that if one requires the existence of a mass gap in the KK graviton spectrum along with the localization of 4D gravity, then the 5D manifold necessarily develops naked singularities at its boundaries. Conversely, if one imposes a smooth character of the Ricci scalar together with 4D gravity localization, then the quantum mechanical potential asymptotically vanishes, a fact that rules out the presence of a mass gap in the graviton spectrum of KK excitations. In this work we include a positive bulk cosmological constant $\Lambda_{5}$ together with an induced flat FRW metric (of de Sitter type, in particular) on the 3-brane.

It is easy to show that for the setup (1) with the ansatz (17) and for the potential $V_{Q M}$ considered in (25), for smooth configurations at the position of the branes we can write the following relation

$$
R \Psi_{0}^{\frac{4}{3}} \sim\left[3\left(\frac{\ddot{a}}{a}+\frac{\dot{a}^{2}}{a^{2}}\right)-\frac{8}{3} V_{Q M}\right]
$$


which apart from the quantities included in [14], it involves the scale factor. Thus, from this expression we see that if one requires the presence of a mass gap in the graviton spectrum of KK excitations, the potential $V_{Q M}$ must asymptotically adopt a positive value; if indeed one requires $4 \mathrm{D}$ gravity to be localized, which means that $\Psi_{0} \longrightarrow 0$ as $z \longrightarrow \infty$, then the Ricci scalar can be either finite or singular depending on the asymptotic behaviour of the expression in brackets: if it asymptotically vanishes, then the Ricci scalar can be finite along the fifth dimension, while if it asymptotically tends to a finite or infinite value, then the curvature scalar will possess naked singularities at the boundaries of the manifold. A similar result was recently obtained in [20] for $\mathrm{AdS}_{5}$ bulk geometries with both dS and AdS induced metrics on the 3-brane, where some examples are displayed as clarifying illustrations.

In our case the scale factor has the particular form corresponding to a de Sitter metric $a=e^{H t}$, yielding

$$
R \Psi_{0}^{\frac{4}{3}} \sim\left[3 H^{2}-\frac{4}{3} V_{Q M}\right]
$$

since the quantum mechanical potential $V_{Q M}$ asymptotically approaches the positive value $9 H^{2} / 4$, ensuring the presence of a mass gap in the graviton spectrum of KK fluctuations, then the expression in brackets asymptotically vanishes, implying that the scalar curvature is regular along the fifth dimension, which is indeed the case since it is constant $R=10 \Lambda_{5} / 3$.

Thus, this result guarantees the smoothness of our braneworld obtained without the use of scalar fields even in the presence of a mass gap in the spectrum of KK graviton fluctuations, an important phenomenological fact that rules out the existence of the dangerous arbitrarily light KK excitations.

\section{The solution as a limit of scalar thick branes}

The above presented solution (7) can also be obtained as a special case or limit of scalar field thick branes, with a bent de Sitter 4d metric, by fixing one parameter of these solutions.

By considering the action of a bulk scalar field $\phi$ with a self-interacting potential $V(\phi)$ minimally coupled to $5 \mathrm{~d}$ gravity

$$
S_{5}=\int d^{5} x \sqrt{|G|}\left[\frac{1}{4} R_{5}-\frac{1}{2}(\nabla \phi)^{2}-V(\phi)\right]
$$

and assuming a de Sitter 3-brane in the metric ansatz (3), one gets the following solution reported by Gremm [8] (see also [7],[15]-[16])

$$
\begin{array}{r}
e^{2 A}=\cos ^{2}(c \sigma), \quad \phi=\frac{1}{c} \sqrt{\frac{3}{2}\left(c^{2}-H^{2}\right)} \ln \left[\frac{1+\tan \left(\frac{c \sigma}{2}\right)}{1-\tan \left(\frac{c \sigma}{2}\right)}\right], \\
V(\phi)=\frac{3}{4} \cosh ^{2}\left(\frac{c \phi}{\sqrt{\frac{3}{2}\left(c^{2}-H^{2}\right)}}\right)\left[3 H^{2}+c^{2}-4 c^{2} \tanh ^{2}\left(\frac{c \phi}{\sqrt{\frac{3}{2}\left(c^{2}-H^{2}\right)}}\right)\right],
\end{array}
$$


where $c$ is an arbitrary constant. The $5 \mathrm{~d}$ manifold with this choice of $A(\sigma)$ has naked singularities at $\sigma= \pm \pi /(2 c)$ and the scalar field diverges at this singular point. However, in the particular case $c=H$, the scalar field vanishes and the potential becomes constant, yielding the action (1) with the corresponding solution (7).

It should be mentioned that when the author of [8] analyses the dynamics of metric fluctuations, he does not properly define the mass in a $4 \mathrm{~d}$ de Sitter spacetime and, consequently, the corresponding Schrödinger equation possesses an analog quantum mechanical potential which vanishes asymptotically when $c=H$, however, he correctly reproduces the pair of bound states present in the model for this limit.

On the other hand, Wang [15] has obtained a family of solutions for the same field system (37), which was borrowed from a $4 \mathrm{~d}$ solution presented in [21], and in terms of the $z$ coordinate reads:

$$
A=-n \ln [\cosh (c z)], \quad \phi=\phi_{0} \sin ^{-1}[\tanh (c z)], \quad V(\phi)=V_{0} \cos ^{2(1-n)}\left(\frac{\phi}{\phi_{0}}\right),
$$

where $\phi_{0}=\sqrt{3 n(1-n)}, V_{0}=n c^{2}[3(1+3 n)] / 2, c$ and $n$ are arbitrary constants and the latter is subject to $0<n<1$.

Because of this inequality, in principle, this family of solutions does not contain our particular solution as a special case, but as a limit $n \rightarrow 1$, when the scalar field vanishes and the potential becomes constant.

The $5 \mathrm{~d}$ curvature scalar for this solution reads

$$
R=4 c^{2} n(3 n+2) \cosh ^{2(n-1)} c z
$$

which is regular for $n \leq 1$ and diverges for $n>1$; it is worth noticing that it becomes constant in the limit $n \rightarrow 1$, a fact which agrees with the analysis performed in the previous section regarding the singular or regular character of the $5 \mathrm{~d}$ manifold.

Finally, it is worth noticing that corrections to Newton's law were not computed either in [8] nor in [15].

\section{Acknowledgements}

The authors acknowledge fruitful and illuminating discussions with U. Nucamendi. This research was supported by grants CIC-4.16 and CONACYT 60060-J. DMM and RRML acknowledge PhD grants from CONACYT and UMSNH. AHA thanks SNI for support.

\section{References}

[1] K. Akama, Pregeometry, Lec. Notes Phys. 176 (1982) 267; V.A. Rubakov and M.E. Shaposhnikov, Extra space-time dimensions: towards a solution of the cosmological constant problem, Phys. Lett. B125 (1983) 139; E.J. Squires, Dimensional reduction 
caused by a cosmological constant, Phys. Lett. B167 (1986) 286; M. Gogberashvili, Four dimensionality in noncompact Kaluza-Klein model, Mod. Phys. Lett. A14 (1999) 2025; A. Brandhuber and K. Sfetsos, Nonstandard compactifications with mass gaps and Newton's law, JHEP 10 (1999) 013.

[2] I. Antoniadis, A Possible new dimension at a few TeV, Phys. Lett. B246 (1990) 377; N. Arkani-Hamed, S. Dimopoulos and G. Dvali, The hierarchy problem and new dimensions at a millimeter, Phys. Lett. B429 (1998) 263; I. Antoniadis, N. ArkaniHamed, S. Dimopoulos and G. Dvali, New dimensions at a millimeter to a Fermi and superstrings at a TeV, Phys. Lett. B436 (1998) 257.

[3] M. Gogberashvili, Hierarchy problem in the shell universe model, Int. J. Mod. Phys. D11 (2002) 1635; Localizing gravity in extra dimensions, Eur. Phys. J. C47 (2006) 857.

[4] L. Randall and R. Sundrum, An alternative to compactification, Phys. Rev. Lett. 83 (1999) 4690; A large mass hierarchy from a small extra dimension, Phys. Rev Lett. 83 (1999) 3370; J. Lykken and L. Randall, The shape of gravity, JHEP 06 (2000) 014 .

[5] I.P. Neupane, Accelerating universe from warped extra dimensions, Class. Quant. Grav. 26 (2009) 195008; Extra dimensions, warped compactifications and cosmic acceleration, Phys. Lett. B683 (2010) 88; The Warping of extra spaces accelerates the expansion of the universe, arXiv:1004.0254 [gr-qc].

[6] J. Garriga and T. Tanaka, Gravity in the brane world, Phys. Rev. Lett. 84 (2000) 2778; A. Kehagias and K. Tamvakis, Localized gravitons, gauge bosons and chiral fermions in smooth spaces generated by a bounce, Phys. Lett. B504 (2001) 38; R. Maartens, Brane world gravity, Living Rev. Rel. 7 (2004) 07; L.A. Gergely and R. Maartens, Asymmetric brane-worlds with induced gravity, Phys. Rev. D71 (2005) 024032 .

[7] O. De Wolfe, D.Z. Freedman, S.S. Gubser and A. Karch, Modeling the fifth dimension with scalars and gravity, Phys. Rev. D62 (2000) 046008.

[8] M. Gremm, Four-dimensional gravity on a thick domain wall, Phys. Lett. B478 (2000) 434; Thick domain walls and singular spaces, Phys. Rev. D62 (2000) 044017.

[9] C. Csaki, J. Erlich, T. Hollowood and Y. Shirman, Universal Aspects of gravity localized on thick branes, Nucl. Phys. B581 (2000) 309.

[10] A. Melfo, N. Pantoja and A. Skirzewski, Thick domain wall space-time with and without reflection symmetry, Phys. Rev. D67 (2003) 105003; D. Bazeia, F.A. Brito and A.R. Gomes, Locally localized gravity and geometric transitions, JHEP 11 (2004) 070; O. Castillo-Felisola, A. Melfo, N. Pantoja and A. Ramirez, Localizing gravity 
on exotic thick three-branes, Phys. Rev. D70 (2004) 104029; D. Bazeia, A.R. Gomes and L. Losano, Gravity localization on thick branes: a numerical approach, Int. J. Mod. Phys. A24 (2009) 1135.

[11] O. Arias, R. Cardenas and Israel Quiros, Thick brane worlds arising from pure geometry, Nucl. Phys. B643 (2002) 187; N. Barbosa-Cendejas and A. Herrera-Aguilar, 4D gravity localized in non $Z_{2}$-symmetric thick branes, JHEP 10 (2005) 101; N. BarbosaCendejas and A. Herrera-Aguilar, Localization of $4 \mathrm{D}$ gravity on pure geometrical thick branes, Phys. Rev. D73 (2006) 084022; Erratum-ibid. 77 (2008) 049901; Y.-X. Liu, X.-H. Zhang, L.-D. Zhang, and Y.-S. Duan, Localization of Matters on Pure Geometrical Thick Branes, JHEP 02 (2008) 067.

[12] N. Barbosa-Cendejas, A. Herrera-Aguilar, M.A. Reyes and C. Schubert, Mass gap for gravity lozalized on Weyl thick branes, Phys. Rev. D77 (2008) 126013; N. BarbosaCendejas, A. Herrera-Aguilar, U. Nucamendi and I. Quiros, Mass hierarchy and mass gap on thick branes with Poincare symmetry, arXiv:0712.3098 [hep-th]; N. BarbosaCendejas, A. Herrera-Aguilar, U. Nucamendi, I. Quiros, M. A. Reyes Santos and C. Schubert, Mass gap for gravity localized on thick branes, AIP Conf. Proc. 1083 (2008) 5 .

[13] D. Bazeia, F.A. Brito and J.R. Nascimento, Supergravity brane worlds and tachyon potentials, Phys. Rev. D68 (2003) 085007; R. Koley and S. Kar, Scalar kinks and fermion localisation in warped spacetimes, Class. Quantum Grav. 22 (2005) 753; C. Bogdanos, A. Dimitriadis and K. Tamvakis, Brane models with a Ricci-coupled scalar field, Phys. Rev. D74 (2006) 045003; V. Dzhunushaliev, Thick brane solution in the presence of two interacting scalar fields, Grav. Cosmol. 13 (2007) 302; C. Bogdanos and K. Tamvakis, Brane Cosmological Evolution With Bulk Matter, Phys. Lett. B646 (2007) 39; C.A.S. Almeida, M.M. Ferreira, Jr., A.R. Gomes and R. Casana, Fermion localization and resonances on two-field thick branes, Phys. Rev. D79 (2009) 125022; V. Dzhunushaliev, V. Folomeev, D. Singleton and S. AguilarRudametkin, 6D thick branes from interacting scalar fields, Phys. Rev. D77 (2008) 044006; M. Gogberashvili, D. Singleton, Nonsingular Increasing Gravitational Potential for the Brane in 6D, Phys. Lett. B582 (2004) 95; K. Farakos, G. Koutsoumbas and P. Pasipoularides, Graviton localization and Newton's law for brane models with a non-minimally coupled bulk scalar field, Phys. Rev. D76 (2007) 064025.

[14] A. Herrera-Aguilar, D. Malagon-Morejon, R.R. Mora-Luna, U. Nucamendi, Aspects of thick brane worlds: 4D gravity localization, smoothness, and mass gap, Mod. Phys. Lett. A25 (2010) 2089.

[15] A. Wang, Thick de Sitter 3 branes, dynamic black holes and localization of gravity, Phys. Rev. D66 (2002) 024024. 
[16] S. Kobayashi, K. Koyama and J. Soda, Thick Brane Worlds and Their Stability, Phys. Rev. D65 (2002) 064014.

[17] M.K. Parikh and S.N. Solodukhin, De Sitter brane gravity: From close-up to panorama, Phys. Lett. B503 (2001) 384; M. Minamitsuji, W. Naylor and M. Sasaki, Quantum fluctuations on a thick de Sitter brane, Nucl. Phys. B737 (2006) 121; S. Pal and S. Kar, de Sitter branes with a bulk scalar, Gen. Rel. Grav. 41 (2009) 1165; X.-H. Zhang, Y.-X. Liu, and Y.-S. Duan, Localization of Fermionic Fields on Braneworlds with Bulk Tachyon Matter, Mod. Phys. Lett. A23 (2008) 2093; Y.-X. Liu, J. Yang, Z.-H. Zhao, C.-E. Fu, and Y.-S. Duan, Fermion Localization and Resonances on A de Sitter Thick Brane, Phys. Rev. D80 (2009) 065019; Y.-X. Liu, C.-E. Fu, L. Zhao, and Y.-S. Duan, Localization and Mass Spectra of Fermions on Symmetric and Asymmetric Thick Branes, Phys. Rev. D80 (2009) 065020; Y.-X. Liu, Z.-H. Zhao, S.-W. Wei and Y.-S. Duan, Bulk Matters on Symmetric and Asymmetric de Sitter Thick Branes, JCAP 02 (2009) 003; Y.-X. Liu, H. Guo, C.-E. Fu, and J.-R. Ren, Localization of Matters on Anti-de Sitter Thick Branes, JHEP 02 (2010) 080.

[18] R.M. Wald, General Relativity, University of Chicago Press, Chicago (1984) 491 pp.

[19] C. Gabriel and P. Spindel, Massive spin-2 propagators on de Sitter space, J. Math. Phys. 38 (1997) 622; T. Garidi, What is mass in de Sitterian physics? hepth/0309104; J. P. Gazeau and M. Novello, The question of Mass in (anti-) de Sitter Spacetimes, J. Phys. A41 (2008) 304008.

[20] H. Guo, Y.-X. Liu, S.-W. Wei and C.-E. Fu, Aspects of Bent Thick Brane Worlds: Gravity Localization and 5D Curvature Scalar, arXiv:1008.3686 [hep-th].

[21] G. Goetz, The gravitational field of plane symmetric thick domain walls, J. Math. Phys. 31 (1990) 2683. 